\title{
Subjective Bayesian analysis of the elliptical model
}

\author{
J. van Niekerk ${ }^{1}$, A. Bekker, M. Arashi and J.J.J. Roux \\ Department of Statistics, Faculty of Natural and Agricultural Science, University of \\ Pretoria, Pretoria, South Africa
}

\section{Abstract}

The multivariate elliptical model is considered, such as to derive subjective Bayesian estimators of the location vector and some functions of the characteristic matrix for the normal-inverse Wishart prior and the normal-Wishart prior which was considered by Bekker and Roux (1995). Fang and Li (1999) considered the elliptical model for Bayesian analysis but with an objective prior structure.

Keywords: Bayesian, characteristic matrix, elliptically contoured, location vector, normalinverse Wishart, normal-Wishart.

\section{Introduction}

The multivariate normal distribution is a well-known and widely discussed distribution for which a large amount of literature exists. It is however necessary to extend the results that does exist for a more general case such as the elliptical model since there are a lot of natural phenomena, especially in the finance and risk sectors, where the normal model is inadequate as a modelling distribution. The elliptically contoured distribution (Fang and Zhang, 1990) can be viewed as an extension of the multivariate normal distribution and hence possesses some of the same properties such as symmetry. An elliptically contoured distribution is a distribution whose contours of equal density have the same elliptical shape as that of the normal distribution but it can also be long-tailed or short-tailed. This type of distribution is therefore more flexible than the normal model and hence its increase in popularity. Some examples of an elliptically contoured distribution is the Pearson type VII distributions of which the multivariate Student t-distribution forms part and the generalized Laplace and Bessel distributions, to name but a few. Elliptically contoured distributions has been investigated from as early as 1860 by Maxwell, amongst others, and have since then received a large amount of interest from modern researchers such as Fang and Zhang (1990) and Gupta and Varga (1993). The contributions made by Díaz-García and Vera (2011), Gómez et al (2003), Cheung et al (2007) and Arashi and Tabatabaey (2008) should be acknowledged.

The objective of this paper is to derive Bayes estimators for the parameters of the multivariate elliptically contoured distribution by using subjective Bayesian analysis. Fang and $\mathrm{Li}$ (1999) considered the elliptical matrix model for Bayesian analysis but with an objective prior structure. Subjective analysis generally produces more admissible results since added information is used than in the case of objective analysis. None the less,

\footnotetext{
${ }^{1}$ Corresponding author: Fax +27 12420 3440 E-mail address: Janet.vanNiekerk@up.ac.za (J. van Niekerk)
} 
very few results and estimators for subjective Bayesian analysis exist and this presentation attempts to contribute to the literature in terms of more general subjective Bayesian estimation.

For a discussion of the Bayesian analysis of the multivariate normal model see Press (1982) with the assumption of a diffuse prior and also a conjugate prior in the form of the normal model for the location parameter and the inverse Wishart distribution for the covariance matrix. The normal-inverse Wishart prior is known as the natural conjugate prior for the multivariate normal model because of the mathematical tractability in the derivation of the posterior distributions and the Bayes estimator. In many cases mathematical ease is not the goal but rather a prior distribution that maximally reflects the prior information and it is for this reason that a normal-Wishart prior, as discussed by Bekker and Roux (1995), will also be considered in this presentation to optimally consume the prior information.

For the purpose of this presentation we give the definition of a univariate and multivariate elliptically contoured distribution as follows:

Definition 1 (Rao, 1990). The univariate elliptically contoured distribution is defined for a random variable $X$ if the density function of $X$ is given by

$$
f\left(x \mid \mu, \sigma^{2}\right)=c \sigma^{-1} g\left[\left(\frac{x-\mu}{\sigma}\right)^{2}\right]
$$

The function $g: \mathbb{R}^{+} \rightarrow \mathbb{R}^{+}$is called the density generator and it is a function of the quadratic form $\left(\frac{x-\mu}{\sigma}\right)^{2}$. It is denoted as $X \sim \operatorname{ECD}\left(\mu, \sigma^{2}, g\right)$.

Definition 2 (Fang $\&$ Zhang,1990). A random vector $\mathbf{X} \in \mathbb{R}^{p}$ has a multivariate elliptically contoured distribution (ECD) with parameters $\boldsymbol{\mu}, \boldsymbol{\Sigma}$ and $g$ if its density function is

$$
f(\mathbf{x} \mid \boldsymbol{\mu}, \boldsymbol{\Sigma})=d_{p}|\boldsymbol{\Sigma}|^{-\frac{1}{2}} g\left[\frac{1}{2}(\mathbf{x}-\boldsymbol{\mu})^{\prime} \boldsymbol{\Sigma}^{-1}(\mathbf{x}-\boldsymbol{\mu})\right], \boldsymbol{\mu} \in \mathbb{R}^{p}, \boldsymbol{\Sigma}>\mathbf{0}
$$

where $d_{p}=\frac{\Gamma\left(\frac{1}{2 \pi}\right)}{\left(\frac{1}{2 \pi}\right)^{\frac{p}{2}}}\left(\int_{\mathbb{R}^{p}} y^{p-1} g\left(y^{2}\right) d y\right)^{-1}$ with $\Gamma(\cdot)$ the gamma function. The function $g$ : $\mathbb{R}^{+} \rightarrow \mathbb{R}^{+}$is called the density generator and it is a function of the quadratic form $\frac{1}{2}(\mathbf{x}-\boldsymbol{\mu})^{\prime} \boldsymbol{\Sigma}^{-1}(\mathbf{x}-\boldsymbol{\mu})$. It is denoted as $\mathbf{X} \sim \operatorname{ECD}(\boldsymbol{\mu}, \boldsymbol{\Sigma}, g)$ with $\boldsymbol{\mu}$ as the location vector and $\boldsymbol{\Sigma}$ as the characteristic matrix.

Therefore in this paper, the main focus is on subjective aspects regarding the Bayesian paradigm, with the multivariate elliptical model as the underlying distribution. The normal-inverse Wishart joint prior distribution and normal-Wishart joint prior distribution are assumed respectively, for the location vector and characteristic matrix of this underlying model. The joint posterior density functions, marginal posterior density functions and Bayesian estimators of the parameters are derived. In this study it is shown that the Bayesian estimator of the location parameter is a robust estimator in the sense that it is independent of the prior distribution of the scale parameter and the form of the density of the specific elliptical model in consideration. This is a very good result since this estimator can then be easily applied to all elliptical models. Lastly, the methodology will be illustrated by a real data set. This paper makes a substantial contribution to the field of modern multivariate analysis with the implementation of the elliptically contoured models form subjective prior view. 


\section{Acknowledgements}

The authors would like to hereby acknowledge the support of the StatDisT group. This work is based upon research supported by the National Research foundation, Grant (Re:2007043000003) and The Research and Development Programme, University of Pretoria.

\section{References}

1. Arashi, M. and Tabatabaey, S.M.M. (2008). Estimation in multiple regression model with elliptically contoured errors under MLINEX loss, Journal of Applied Probability and Statistics, 3(1):23-35.

2. Bekker, A. and Roux, J.J.J. (1995). Bayesian multivariate normal analysis with a Wishart prior, Communications in Statistics-Theory and Methods, 24(10):24852497.

3. Cheung I,W., Wong, H. and Liu, J.S. (2007). Inverse Wishart distributions based on singular elliptical contoured distributions, Linear Algebra and its Applications, 420:424-432.

4. Díaz-García, J.A. and Vera, J.F. (2011). Multivariate three-parameter log-elliptical distributions, Communications in Statistics-Theory and Methods, 40(1):1964-1976.

5. Fang, K.T, and Li, R.Z. (1999). Bayesian statistical inference on elliptical matrix distributions, Journal of multivariate analysis, 70:66-85.

6. Fang, K.T. and Zhang, Y. (1990). Generalized Multivariate Analysis. SpringerVerlag and Science Press, Berlin and Beijing.

7. Gómez, E., Gómez-Villegas, M.A. and Marin, J.J. (2003). A survey on continuous elliptical vector distributions, Revista Matemática, 16(1):345-361.

8. Gupta, A.K. and Varga, T. (1993). Elliptically contoured models in Statistics. Kluwer Academics, Dordrecht.

9. Press, S.J. (1982). Applied Multivariate Analysis. Holt, Rinchart \& Winston, New York.

10. Rao, B.L.S.P. (1990). Remarks on univariate elliptical distributions, Statistics and Probability Letters, 10:307-315. 